\title{
Titanium dioxide photocatalysis for pharmaceutical wastewater treatment
}

\author{
Devagi Kanakaraju • Beverley D. Glass • \\ Michael Oelgemöller
}

Received: 22 May 2013/Accepted: 27 May 2013/Published online: 6 June 2013

(C) Springer-Verlag Berlin Heidelberg 2013

\begin{abstract}
Heterogeneous photocatalysis using the semiconductor titanium dioxide $\left(\mathrm{TiO}_{2}\right)$ has proven to be a promising treatment technology for water purification. The effectiveness of this oxidation technology for the destruction of pharmaceuticals has also been demonstrated in numerous studies. This review highlights recent research on $\mathrm{TiO}_{2}$ photocatalytic treatment applied to the removal of selected pharmaceuticals. The discussions are tailored based on the therapeutic drug classes as the kinetics and mechanistic aspects are compound dependent. These classes of pharmaceuticals were chosen because of their environmental prevalence and potential adverse effects. Optimal operational conditions and degradation pathways vary with different pharmaceutical compounds. The main conclusion is that the use of $\mathrm{TiO}_{2}$ photocatalysis can be considered a state-of-the-art pharmaceutical wastewater treatment methodology. Further studies are, however, required to optimize the operating conditions for maximum degradation of multiple pharmaceuticals in wastewater under realistic conditions and on an industrial scale.
\end{abstract}

Keywords Advanced oxidation processes . Photocatalysis - Titanium dioxide · Pharmaceuticals . Wastewater · Water treatment $\cdot$ Non-steroidal anti-inflammatory drugs · Analgesics · Antibiotics . Antiepileptics

D. Kanakaraju · B. D. Glass · M. Oelgemöller $(\bowtie)$

School of Pharmacy and Molecular Sciences, James Cook

University, Townsville, QLD 4811, Australia

e-mail: michael.oelgemoeller@jcu.edu.au

\section{Introduction}

Poor sanitation, water scarcity, deterioration of water quality, emergence of waterborne diseases, and lack of clean water supply are all posing global challenges due to the rising demand by an increasing world population. Further demands on water resources have resulted from the introduction of emerging recalcitrant contaminants or xenobiotics, such as pharmaceuticals, endocrine disruptors, surfactants, and personal care products, which are complex and resistant to degradation. The number of studies highlighting the prevalence of pharmaceuticals in water environments has increased significantly since 2000, Heberer (2002), Tixier et al. (2003), Fent et al. (2006), and Miège et al. (2009). This has raised widespread concern about the lack of knowledge of potential effects of pharmaceutical compounds and their metabolites on human and aquatic organisms, despite occurring in trace quantities ranging from $\mathrm{ng} / \mathrm{L}$ to $\mu \mathrm{g} / \mathrm{L}$, Kümmerer (2009) and Mompelat et al. (2009).

Existing sewage treatment technology is considered inadequate for the removal of emerging micropollutants, as they are not designed to handle this specific class of pollutants, Suárez et al. (2008). The failure of conventional wastewater treatment plants (WWTPs) to remove pharmaceuticals clearly shows the urgent need for innovative technologies that can effectively deal with these compounds, Ternes (1998) and Zhang et al. (2008a). Advanced oxidation processes (AOPs) are regarded as appropriate to degrade pollutants, which are known to be non-biodegradable or have low biodegradability, persistence, and possess high chemical stability. AOPs for water and wastewater treatment include photolysis and photocatalysis, ozonation, Fenton and photo-Fenton, ultrasound radiation, sonolysis, electrochemical oxidation, and wet air oxidation. 